\title{
Neue Therapie-Optionen für Melanom-Patienten
}

Innerhalb von wenigen Jahren haben sich die Möglichkeiten für Patienten mit metastasiertem Melanom deutlich zum Positiven gewandelt. Auch in den nächsten Jahren ist eine Vielzahl von neuen Behandlungsoptionen für verschiedene Gruppen von Melanom-Patienten zu erwarten.

\begin{abstract}
„Die Therapien zur Behandlung von Hautkrebs haben sich in jüngsterVergangenheit eindrucksvoll verändert und verbessert. Die früher übliche Chemotherapie ist in vielen Bereichen inzwischen nur mehr die letzte Option. Die heutige Standardtherapien ruhen auf zwei Säulen: der molekular gezielten Therapie und der Immuntherapie", erläuterte Kongresspräsident Prof. Dr. Christoph Höller von der Klinischen Abteilung für Allgemeine Dermatologie und Dermato-Onkologie am AKH Wien anlässlich des 12. Congress of the European Association of Dermato-Oncology (EADO).
\end{abstract}

\section{Molekular gezielte Therapien}

Bei bis zu 50\% der Melanom-Patienten wird eine Mutation im BRAF-Gen gefunden. Das BRAF-Protein ist wichtig für das Wachstum und das Überleben von Zellen, in seiner mutierten Form kann es jedoch zu unkontrolliertem Wachstum und somit auch zu einer Krebserkrankung führen. Höller: „Die Entdeckung der BRAF-Mutation ist einer der wichtigsten Meilensteine für die MelanomBehandlung. Sie hat die Hautkrebstherapie von Grund auf revolutioniert und modernisiert."

\section{Immuntherapie stärkt das Abwehrsystem}

Bei der Immuntherapie wird nicht der Tumor selbst bekämpft, sondern es wird das körpereigene Abwehrsystem gestärkt. Diese Therapieform konnte dank der Entdeckung von „Immune Checkpoints" entwickelt werden: Rezeptoren, welche die Aktivierung beziehungsweise die Hemmung von Abwehrzellen wie etwa T-Lymphozyten übertragen. Immun-Checkpoint-Inhibitoren blockieren gezielt hemmende Rezeptoren. Höller:„Insbesondere mit den
PD-1-Antikörpern liegen derzeit effektive und gut verträgliche Substanzen vor, die auch in den kommenden Jahren für Einzeltherapien oder Kombinationstherapien bei metastasierten Melanomen eingesetzt werden können."

Derzeit wird auch darüber diskutiert, die moderne Immuntherapie bei Patienten einzusetzen, die trotz einer kompletten Operation ein sehr hohes Rückfalls-Risiko haben. Höller: „Davon erhofft man sich eine höhere Wirksamkeit und eine bessere Verträglichkeit als bei einer Chemotherapie mit dem bisher verwendeten Interferon." Die neuen Therapien werden nicht nur beim Melanom angewandt, sondern auch bei sehr weit fortgeschrittenen Formen von Weißem Hautkrebs oder bei seltenen Hauttumoren wie dem Merkelzellkarzinom. Höller weiter:„,Die modernen Immuntherapien zeichnen sich besonders durch das bei einem Teil der Patienten beobachtete verbesserte Langzeit-Überleben aus. Derzeit sind die neuen Therapien aber noch nicht bei allen Formen von Hautkrebs etabliert."

\section{Therapiekombinationen}

„Unterschiedliche Immuntherapien und gezielte molekulare Therapien können auch parallel eingesetzt werden, um die Wirksamkeit weiter zu erhöhen", erklärte Höller. Manche Kombinationen sind bereits zugelassen oder sogar schon zum neuen Standard geworden. Gegenwärtig werden auch sehr viele verschiedene Kombinationsmöglichkeiten in Studien erprobt. „Für Patientinnen und Patienten mit inoperablen Metastasen wird beispielsweise intensiv an einer Kombinationstherapie gearbeitet, die wirksamer und nebenwirkungsärmer ist. In den nächsten Jahren ist eine Vielzahl von neuen Therapie-Optionen für verschiedene Gruppen von Melanom-Patienten zu erwarten."

\section{Schonendere Operationen}

Neue Erkenntnisse sorgen inzwischen auch für einen schonenderen Umgang mit Hautkrebspatienten bei Operationen: In den 1990-er Jahren wurde die Wächter-Lymphknotenbiopsie zur Früherkennung eingeführt. Damit kann bei etwa $20 \%$ der Hautkrebs-Patienten eine Mikrometastasierung festgestellt werden. Bislang galt: Bei Mikrometastasierung muss eine komplette Lymphknotenausräumung vorgenommen werden.

Bei einer kürzlich in Deutschland durchgeführten Studie mit 480 Patienten wurde nur bei der Hälfte der Patienten eine Lymphknotenausräumung vorgenommen, die andere Hälfte erhielt nur Nachsorgeuntersuchungen mit Beobachtung. Die Lymphknoten wurden nur dann ausgeräumt, wenn sich im Lymph-Abflussbassin erneut Metastasierungen bildeten. [1] „Der Unterschied zwischen den beiden Patientengruppen bezüglich der Überlebensrate lag bei weniger als einem Prozent", so Prof. Dr. Claus Garbe, Ärztlicher Leiter der Sektion für Dermato-Onkologie der Universitäts-Hautklinik Tübingen. „Das bedeutet, man kann Melanom-Patienten diesen Eingriff ersparen, wenn die Lymphknoten-Mikrometastasen kleiner als ein Millimeter sind."

\section{Literatur}

\footnotetext{
1. Leiter et al (2016) Complete lymph node dissection versus no dissection in patients with sentinel lymph node biopsy positive melanoma (DeCOG-SLT): a multicentre, randomised, phase 3 trial. Lancet Oncol:1 -11
}

hautnah $2016 \cdot 15: 98$

DOI 10.1007/s12326-016-0216-2

Online publiziert: 13. Oktober 2016

(c) Springer-Verlag Wien 2016 